\section{Receptors and recognition}

Receptors and Recognition. Series A. Vol. 5. Edited by P. Cuatrecasas and M. F. Greaves. Pp. 212. (Chapman and Hall: London, 1978.) Hardback £10; paperback $£ 6$.

Previous volumes in this series have contained four or five articles, but this one contains only three, albeit rather longer, contributions. "Relationships in the structure and function of receptors for glycoprotein hormones, bacterial toxins and interferon" by L. D. Kohn is a contribution central to the theme of the series, whereas "Stereoselective recognition in biology" by $\mathbf{P}$. A. Lehmann and "Fluorescence and NMR studies of membranes" by A. G. Lee can be considered to some degree as contributions to the general education of those interested in biological recognition processes.

Lehmann's chapter is a wide-ranging discussion of the ways in which the mutual recognition between macromolecules and small ligands may be influenced by stereochemical variations in the nature of the bound ligand. It draws its examples from an extraordinary diversity of receptor and enzymic recognition systems, thus placing shoulder to shoulder the pheromones of bark beetles, the proteolytic specificity of papain, conformationally hindered thyroxine derivatives and the interaction between avidin and biotin. Not unnaturally, substantial space is given to the author's own 'eudismic' method for deriving quantitative relationships between affinity and stereoselectivity in various series of homologous pairs of stereoisomers.

As there have recently been a considerable number of reviews of the contributions of spectroscopic techniques to understanding of membrane structure, some of them by Lee himself, a chapter on this topic would only seem justified if it emphasised the ways in which such techniques might be helpful to students of biological recognition phenomena. Unfortunately, this is not what is offered. Instead, the chapter opens with good intentions of interpreting NMR data for the uninitiated, but it soon becomes yet another detailed discussion of the spectroscopic characteristics of hydrated lipids, especially dipalmitoylphosphatidylcholine. Proteins, and even receptors, do get briefly discussed in the final section on fluorescence studies, but it seems doubtful whether many general readers will get this far.

Finally, Kohn's contribution takes us to the heart of recent studies on the receptors for a superficially diverse 'family' of ligands (glycoprotein hormones, bacterial toxins and interferon). It is mainly through the work of Kohn and his colleagues, particularly their studies on gangliosides as receptor components and on sequence homology, that these ligands and their receptors can in any sense be regarded as a family. Because of this, the chapter is to be welcomed, but with the slight suspicion that Kohn may, at least sometimes, be adept at seeing unities where none exist. As this is very much the chapter of an enthusiast, it is filled with intriguing snippets of unexplained new information which will keep the

\section{Conformational energy surfaces}

Biomolecular Information Theory: Studies in Physical and Theoretical Chemistry. By S. Fraga, K. M. S. Saxena and M. Torres. Pp. 271. (Elsevier: Amsterdam, Oxford and New York, 1978.) \$48.75; Dfl.112.

READING this book was a horrible experience. I hasten to add that this was not a fault of the book itself, which has many commendable features. It was merely a manifestation of that recurrent nightmare of textbook writers, a new book with similarities to that which he is in the throws of writing. After some deliberation, it seemed that it would be fun to review a book from an unusually informed viewpoint, but the reader of this review is forewarned that the reviewer may be revealing some of the prejudices of a literary cuckold.

Inevitably, closer inspection revealed many important differences. It is primarily an essay in quantum biochemistry, with emphasis on the conformational energy surfaces of units of protein and nucleic acid molecules. The title is, perhaps, somewhat misleading, as the book does not contain much more about biomolecular information than most biochemical and biophysical textbooks, and there is very little information theory proper. Nonetheless, the first major section provides an illuminating discussion of topics peripheral to an information theory of biological molecules, including the mathematics of the prebiotic origins of self-replicating systems. There is also an entertaining account of the formation of amino acids in the prebiotic soup, and of the occurrence of amino acids in meteorites and Moon dust.

The second major section is largely a vehicle for discussing the stereochemical code of proteins and nucleic acids in terms of the conformational behaviour of low molecular weight analogues of their units. In particular, reader's appetite whetted for the next episodes in this intriguing tale.

Overall, therefore, this is an uneven book with too much space devoted to material far from the nitty-gritty of receptors and recognition. Its proofreading may be a bit better than in earlier volumes (see review in Nature 274, 192-193; 1978) but there is stifl no index (purchasers of volume 6, as yet unpublished, are to get the indices of volumes $1-5$ as a bonus). R. H. Michell

R. H. Michell is Lecturer in Biochemistry at the University of Birmingham, UK.

$\mathrm{N}$-acetyl $\mathrm{N}^{\prime}$-methylamide derivatives of amino acids are considered and there is an exhaustive treatment of their conformational energy surfaces as a function of the two major angles of rotation in the backbone. There is also an extensive discussion of the theory and methods (with appendices) for calculating such energy surfaces, and of techniques (such as minimisation and Monte Carlo) for treating more complex energy surfaces. Unfortunately, the authors run the risk of placing too much emphasis on one type of energy calculation, the semi-empirical quantum mechanioal PCILO technique of Maigret and Pullman. Indeed, much of the book reads like an encyclopaedia of PCILO results. This gives the text a certain consistency, but the danger of emphasising one type of calculation is that the future usefulness of the book depends to some extent on the validity of that calculation. It was a little onesided of the authors to opt for PCILO, because the energy surfaces calculated by this method are somewhat anomalous with respect to those derived by the classical studies, as well as by recent empirical crystal-derived potentials and extended basis set $a b$ initio calculations. Some CNDO results are reported, although this is one of the few types of calculation which produces similar energy surfaces to PCILO. The consequence of this is that the stereochemical code as viewed by the authors well allows some conformational features not believed by many workers.

Incidentally, the energy maps are presented in the old angle convention, which might cause some confusion in the partially initiated, particularly as the old convention is not used consistently throughout the book.

More generally, the book is pleasant to read, although the jargon is not well suited to biologists. Its mood is technical rather than journalistic ("Valinomycin is a cyclodecadepsipeptide"). A few sections, such as that on "Topological Characteristics of Energy Hypersurfaces", turned out to be more trivial than suggested by a first read. 